\title{
EFEITOS DOS FILTROS HIGROSCÓPICO E HIDROFÓBICO NO CLEARANCE DO MUCO E BARREIRA DE BACTÉRIAS EM PACIENTES COM DOENÇA HEPÁTICA
}

\author{
Effects of hygroscopic and hydrophobic filters in the mucus clearance and bacterial barriers \\ in patients with liver disease
}

Eliane Maria Carvalho Silva'; Maria Rita Montenegro Isern²; Paulo C. B. Massarollo3; Paulo Hilário Nascimento Saldiva4;
Sérgio Mies ${ }^{5}$ \& Geraldo Lorenzi- Filho

\begin{abstract}
RESUMO
Objeivo: Comparar os efeitos dos filtros hidrofóbico e higroscópico nas propriedades físicas e transportabil idade do muco e na incidência de infecção pulmonar. Casuística eMétoda Foram estudados 35 pacientes com doença hepática, internados na UTI de Transplante de Fígado e submetidos à ventilação mecânica por mais de 24 horas. Os pacientes foram randomizados em dois grupos, imediatamente após a intubação. Grupo 1 ( $n=19)$ usou o filtro hidrofóbico - BB100-(PA LL) e grupo $2(n=16)$ usou o filtro higroscópico - Humid-Vent Filter ${ }^{(}(\mathrm{GIBEC})$. A média de idade dos grupos hidrofóbico e higroscópico era 52,4 13,0 e 45,5 16,4 anos, respectivamente. Resultados 0 s pacientes eram semel hantes em relação à fração inspi rada de oxigênio, à pressão parcial de oxigênio / fração inspi rada oxigênio, ao volume minuto, ao balanço hídrico nas últimas 24 horas e ao valor de sódio e bilirrubina. A transportabilidade do muco foi avaliada através do transporte ciliar e da tosse. A adesão foi medida pelo ângulo do contato. A análise do filtro como barreira de bactéria foi avaliada por cultura da secreção traqueal. Os grupos tiveram resultados similares em relação à adesão, ao transporte ciliar, à freqüência da contaminação do circuito e à infecção pulmonar. Conclusão: A s propriedades físicas do muco ficaram inalteradas com o uso dos dois filtros. No entanto, o transporte através da tosse foi melhor no grupo higroscópico. A mbos os filtros podem ser considerados como barreira de bactérias e podem reduzir a ocorrência de pneumonia relacionada ao ventilador.
\end{abstract}

Descritores muco brônquico, regenerador de calor e umidade, pneumonia nosocomial, ventilação mecânica, transporte ciliar.

\footnotetext{
Grau Acadêmico:

1. Fisioterapeuta do Serviço de Transplante e Cirurgia do Fígado do HC-FMUSP; Professora do Curso de Pós-graduação do Centro Universitário do Triângulo - UNITRI.

2. Fisioterapeuta do Serviço de Transplante e Cirurgia do Fígado do HC-FMUSP; Professora do Centro Universitário São Camilo

3. Médico Assistente - Departamento de Cirurgia - FMUSP.

4. Professor Titular - Departamento de Patologia - FMUSP.

5. Professor Associado - Departamento de Cirurgia - FMUSP.

6. Médico Assistente - Departamento de Pneumologia - FMUSP.
}

Instituições:

Faculdade de Medicina da Universidade de São Paulo, Brasil - Liver Transplantation and Surgery Centro Universitário do Triângulo - UNITRI

Correspondência:

Eliane Maria de Carvalho Silva

UNITRI - Centro Universitário do Triângulo

Av. Nicomedes Alves dos Santos, 4545,

Bairro Gávea, CEP 38411-106, Uberlândia, MG, Brasil

Phone nr. 553432240172

E-mail: barbosinha@unitri.edu.br

Recebido em: 06/08/2005

Aceito em: 06/10/2005

\section{INTRODUÇÃO}

Sabe-se que a via aérea, além de sua função primária como filtro biológico, inibe a entrada de materiais prejudiciais aos pulmões e também fornece o calor e a umidade, com conseqüente saturação do vapor de água. ${ }^{1}$ Pacientes submetidos à ventilação mecânica sof rem alteração na função respi ratória, necessitando um dispositivo artificial para real izar esta função. Os ventiladores mecânicos têm geralmente um sistema de aquecimento do ar, mas não possuem nenhum filtro de ar. ${ }^{2,3}$ A tualmente, existem vários dispositivos com capacidade de preservar a umidade do ar e alguns com propriedades de filtros para partículas. No entanto, existem controvérsias sobre a eficiência destes dispositivos, não somente na função de umidificação, como também, na função de barreira. ${ }^{4}$

0 umidificador dos respiradores produz ar úmido e aquecido, no entanto, é inevitável o condensado de água nos circuitos, propiciando a colonização destes e, posteriormente, do pulmão. A pneumonia relacionada ao ventilador tornou-se freqüente e uma tentativa de evitá-la foi a substituição dos reservatórios pelos regeneradores de calor e umidade. Este estudo compara dois tipos de filtros umidificadores com propriedades 
diferentes: o filtro higroscópico, altamente absorvente, composto de fibras do propileno e dupla face de papel com cloreto do cálcio, e o filtro hidrofóbico, feito do propileno e coberto por papel cerâmico, produzindo um efeito mecânico na passagem da água. 0 objetivo desses filtros é filtrar 0 ar, evitar perda de água e servir como barreira à entrada de microorganismos. ${ }^{5}$

As infecções pulmonares não representam somente custos elevados da internação e uso de antibióticos, ${ }^{6}$ mas também, conduzem freqüentemente à morte. ${ }^{6,7} \mathrm{O} s$ pacientes com doença hepática são mais suscetíveis às infecções oportunistas; uma restrição protéica, assim como outras infecções recorrentes, são adicionadas à severidade da doença ${ }^{7}$ e as infecções respi ratórias são inevitáveis. ${ }^{6,7} \mathrm{Com}$ todas essas particularidades do doente, a função dos regeneradores, como a dos filtros, é a de formar uma barreira bacteriana, sendo importante na prevenção de infecção. 8,9,10

O objetivo deste estudo foi comparar os efeitos dos filtros higroscópico e hidrofóbico, em relação às propriedades físicas e à transportabilidade do muco, além de avaliar sua ef iciência como barreira bacteriana e a incidência da infecção pulmonar, em pacientes com a doença hepática, sem pneumonia no momento da intubação e submetidos à ventilação mecânica por um tempo maior de 24 horas.

\section{Pacientes e Método}

Foram estudados 35 pacientes com doença hepática crônica, prospectivamente. A pesquisa foi aprovada pelo Comitê de Ética do Hospital das Clínicas - FM USP. Os responsáveis pelos pacientes assinaram o termo de consentimento. Os pacientes não tinham diagnóstico de pneumonia, conforme critérios do Centro de Controle das D oenças e Prevenção (CDC $)^{11}$ e ficaram submetidos à ventilação mecânica por um tempo maior que 24 horas. A indicação da intubação foi: encefal opatia hepática (12), hemorragia digestiva alta (sete) e transplante de fígado (16). Esses pacientes não foram classificados no início do estudo por nenhum escore de gravidade, no entanto, 0 valor médio de bilirrubina total para os grupos 1 e 2 foram 10,0 e 15,8 e de uréia 113,1 e 118,6, o que provavel mente os classificaria como mais graves, utilizando-se 0 índice MELD (Model for endstage liver disease). D evido à indicação de internação, todos os pacientes receberam antibióticos, alguns profiláticos nas cirurgias, outros como tratamento de causas extra-pulmonares. Os pacientes foram randomizados aleatoriamente em dois grupos no momento da intubação: Grupo I - 19 pacientes $(52,4 \pm 13,0$ anos) usou filtro hidrofóbico (BB 100M FS Pall - EUA). Grupo II - 16 pacientes $(45,5 \pm 16,4$ anos) usou filtro higroscópico (H umid-Vent Filter Light-Gibec-Sweden), interposto entre o circuito do ventilador e o tubo oro-traqueal. A secreção pulmonar foi coletada em todos os pacientes, através de um sistema fechado de aspiração, imediatamente após a intubação e em dias consecutivos. Os filtros foram trocados diariamente. No momento da troca do filtro, foram coletadas culturas através de swab das extremidades proximal do filtro (próximo ao paciente) e da extremidade distal (próximo ao circuito do respirador). Os parâmetros do $C D C^{11}$ foram usados para o diagnóstico de pneumonia. A pós a intubação, foi coletado o muco e o filtro selecionado foi introduzido. D urante o procedimento de aspiração, não foi instilada água nem solução salina no tubo para não alterar as propriedades do muco. 0 filtro e o sistema de aspiração foram mudados diariamente. 0 circuito do respirador não foi trocado.

Os dados clínicos dos grupos estão na tabela 1. A s variáveis ventilatórias foram anotadas no momento da coleta do muco: fração inspirada oxigênio (FIO2), relação $\mathrm{PaO} 2 / \mathrm{FIO} 2$, volume minuto, balanço hídrico (últimas 24 horas), valor de sódio e bilirrubina.

Tabela 1: Dados clínicos no primeiro dia

\begin{tabular}{lll}
\hline & GRUP0 1 & GRUP0 2 \\
Idade (anos) & $45,5 \pm 16,4$ & $52,4 \pm 13,0$ \\
Sódio (mg/dl) & $132 \pm 9,31$ & $131 \pm 11,19$ \\
Uréia (mg/dl) & $113,12 \pm 82,03$ & $118,62 \pm 87,57$ \\
Bilirrubina (mg/100ml) & $24,8 \pm 12,06$ & $18 \pm 17,88$ \\
Relação Pa02/FI02 (mmHg/\%) & $228 \pm 57$ & $254 \pm 68$ \\
Fl02 (\%) & $130 \pm 63,26$ & $152 \pm 102,22$ \\
Volume Minuto (I/min) & $9 \pm 2,72$ & $9 \pm 4,20$ \\
\hline
\end{tabular}

Legenda: PaO2 - pressão parcial de oxigênio, FlO2 - fração inspirada de oxigênio

0 transporte mucociliar foi avaliado in vitro. 0 transporte da tosse e 0 ângulo do contato foram analisados, caracterizando a adesividade. ${ }^{1,5} \mathrm{~A}$ outra variável era 0 filtro como barreira de bactérias, que foi avaliada com a análise dos agentes microbiológicos encontrados nas extremidades distal e proximal do filtro e na secreção traqueal. Foi considerada contaminação a presença de pelo menos um agente em uma das extremidades do filtro, com ou sem contaminação da secreção traqueal. A presença de microorganismos iguais aos encontrados na secreção traqueal, na extremidade proximal, foi considerada contaminação da extremidade proximal do filtro. Foi considerada insuficiência de barreira sempre que o mesmo microorganismo da secreção traqueal tivesse sido encontrado também na extremidade distal. A contaminação do circuito foi analisada em relação ao número de experimentos, considerando-se experimento isolado cada dia em que o filtro tenha sido trocado. A análise do swab foi feita através do teste de esterilidade.

O utra variável estudada foi a incidência de pneumonia. Os casos de suspeita de pneumonia eram submetidos a broncoscopia e era lavado o bronco alveolar para identificar 0 agente com cultura qual itativa e quantitativa, como procedimento rotinei ro da U TI onde o trabal ho foi desenvolvido. A incidência de pneumonia foi analisada em relação ao número de pacientes.

\section{Análise estatística}

A análise estatística foi feita com o ajuste de curvas do crescimento para cada grupo dos pacientes..$^{12} \mathrm{~A} s$ curvas lineares do crescimento traduziam o comportamento das variáveis 
estudadas durante 0 tempo em que os filtros foram usados. 0 momento da instalação do filtro foi definido como o tempo "zero". A s observações feitas após a intubação, mas antes da colocação do filtro, foram consideradas como co-variáveis do modelo estatístico ( $x$ ). A s curvas do crescimento foram expressas pela seguinte equação: $v d, x=+. d=.\left(x-x^{*}\right)$, onde, $v d, x=$ média da variável para 0 décimo dia e para $x=$ $x^{*} ; \quad=$ coeficiente linear; = coeficiente angular; $d$ = número dos dias após "o tempo zero"; = coeficiente co-variável; $x=$ observação co-variável individual; $x^{*}=$ média simples da co-variável. 0 coeficiente linear representa a média da população no tempo da colocação do filtro, e sempre que $x$ $=\mathrm{x} *$. O coeficiente angular representa a variação diária da média da população da variável. 0 coeficiente representa a variação média do coeficiente linear para cada aumento de uma unidade no x. 0 ajuste de crescimento foi conseguido, com o uso do coeficiente para os dois grupos. A matriz de covariância usada para as observações a respeito do mesmo indivíduo era do tipo auto-regressiva de primeira ordem. Para cada variável, os dois coeficientes lineares dos grupos foram comparados de acordo com os testes baseados na Distribuição de F isher. Sempre que a igual dade foi concluída, um coeficiente comum para ambos os grupos foi estimado, e foi adotado o nível de significância de $5 \%{ }^{12}$

\section{Resultados}

Os grupos são similares na média de idade, na taxa de mortalidade, tempo de intubação e diagnóstico de pneumonia; isto pode ajudar na comparação dos grupos higroscópico e hidrofóbico, como está demonstrado na tabela 2. Os índices da contaminação por microorganismos iguais nas extremidades distal e proximal e a eficiência da barreira de bactérias dos filtros estão demonstrados na tabela 3.0 número de microorganismos iguais na extremidade proximal do filtro hidrofóbico foi significantemente menor $(p=0,004)$ em relação ao filtro higroscópico, também demonstrado na tabela 3 . Não houve diferença estatística nos grupos em relação à contaminação das extremidades, como está demonstrado na tabela 4.

Tabela 2: Demonstra a media da idade, mortalidade, extubação, tempo de intubação e diagnóstico de pneumonia

\begin{tabular}{llll}
\hline & GROUP 1 & GROUP 2 & P \\
Número de pacientes & 19 & 16 & - \\
Idade (média e dp) & $52,4+13,0$ & $45,5+16,4$ & - \\
Mortalidade (\%) & $(42,1 \%)$ & $(43,8 \%)$ & 0,920 \\
Extubação (\%) & $(57,9 \%)$ & $(56,3 \%)$ & 0,925 \\
Tempo de intubação (dias) & $5,38+4,51$ & $5,42+3,89$ & 0,978 \\
Pneumonia (\%) & $3(15.8 \%)$ & $2(12,5 \%)$ & 0,846 \\
\hline
\end{tabular}

RESULTADOS: Análise da barreira de bactérias

Pela análise gráfica do transporte através da tosse e pelas tendências lineares do crescimento dos coeficientes lineares médios em relação ao tempo, é possível dizer que filtro
Tabela 3: Demonstra o índice de contaminação.

\begin{tabular}{llll}
\hline & GROUP 1 & GROUP 2 & P \\
Número total de experimentos & 85 & 75 & - \\
$\begin{array}{l}\text { Número de microorganismos } \\
\text { iguais (proximal) }\end{array}$ & $34(40,0 \%)$ & $47(62,7 \%)$ & 0,004 \\
$\begin{array}{l}\text { Número de microorganismos } \\
\text { iguais (distal) }\end{array}$ & $5(5,9 \%)$ & $2(2,7 \%)$ & 0,686 \\
\hline
\end{tabular}

Tabela 4: Demonstra o número total de microorganismos das extremidades dos filtros.

\begin{tabular}{lllc}
\hline & GROUP 1 & GROUP 2 & P \\
Número total de experimentos & 85 & 75 & - \\
Contaminação da extremidade distal & $9(10,6 \%)$ & $2(2,7 \%)$ & 0,319 \\
Contaminação da extremidade proximal & $62(72,9 \%)$ & $66(88,0 \%)$ & 0,057 \\
\hline
\end{tabular}

higroscópico mantém valores iguais, enquanto que o filtro hidrofóbico apresenta uma tendência di minuída do crescimento linear, no entanto, sem diferença estati sticamente significante no grupo higroscópico, como está demonstrada na figura 1.0 transporte mucociliar através dos cílios e do ângulo de contato não foi estatisticamente significante nos grupos estudados, ambos podendo ser considerados similares (figuras 2 e 3 ).

\section{Discussão}

0 desenvolvimento de métodos da ventilação mecânica tem favorecido extremamente 0 tratamento dos pacientes com

Figura 1. Os perfis médios e o erro padrão para a variável "tosse", com sua estimada curva de crescimento (a relação $x=x^{*}=4,70$ )

\section{TRANSPORTE ATRAVÉS DA TOSSE}

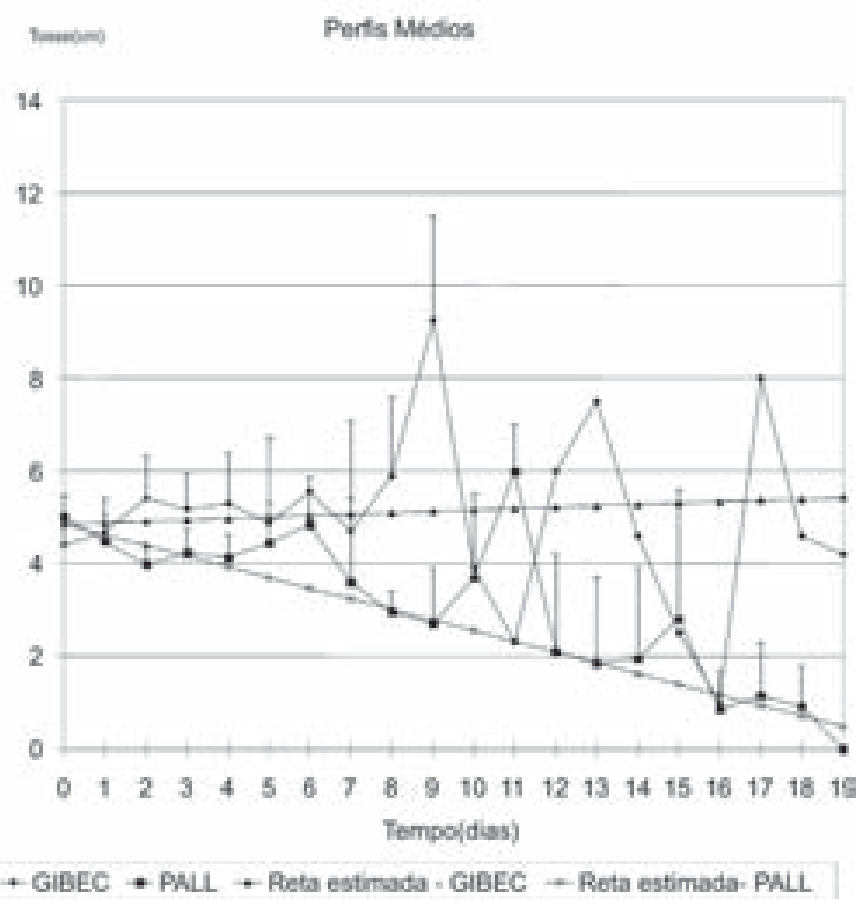


Figura 2. Os perfis médios e o erro padrão para a variável "transporte mucociliar", com sua estimada curva de crescimento (a relação $x=x{ }^{*}=$ 0,88). Não há diferença estatística entre os grupos.

\section{TRANSPORTE MUCOCILIAR}

Perfis médios

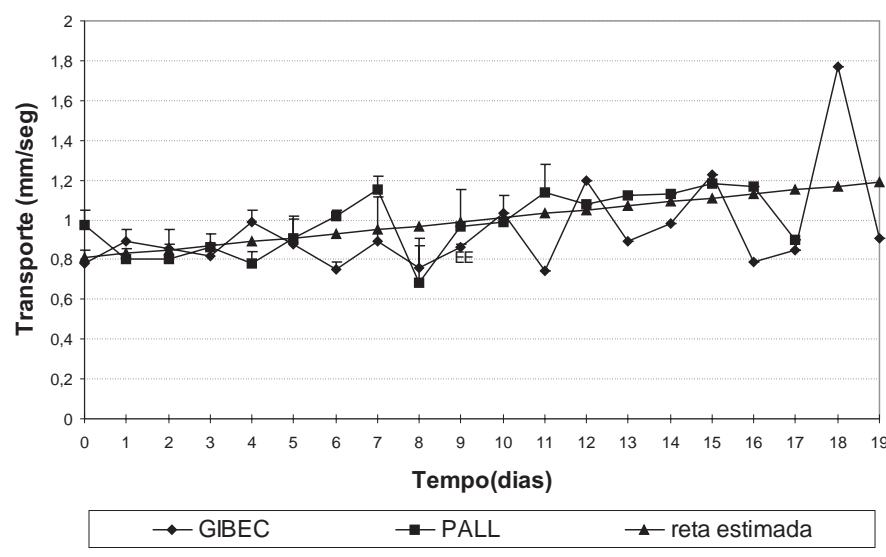

Figura 3: Os perfis médios e o erro padrão para a variável "ângulo de contato", com sua estimada curva do crescimento (a relação $x=x^{*}=31,90$ )

\section{ÂNGULO DE CONTATO}

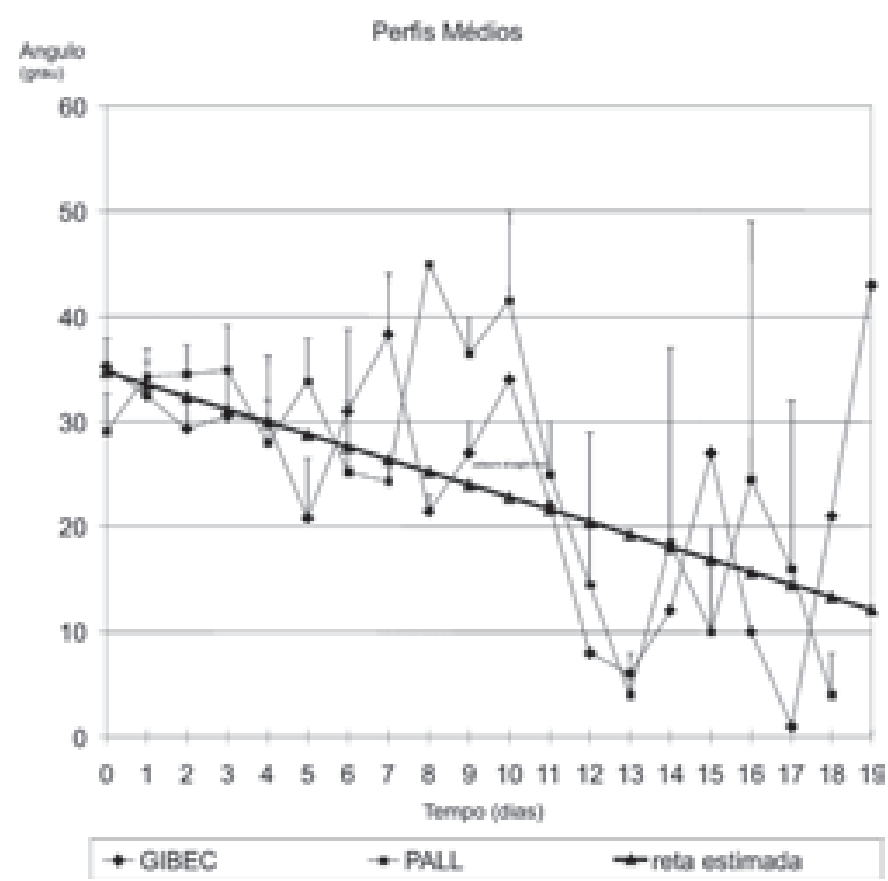

insuficiência respiratória aguda. No entanto, apesar desta evolução, algumas complicações são freqüentes. Desta maneira, é importante fornecer uma orientação contínua à equipe profissional que cuida dos pacientes e manipula 0 respirador, manipulação correta dos cateteres, em relação aos cuidados para evitar que os pacientes aspirem secreções da orofaringe e gástrica, causando auto-infecção. ${ }^{13,14}$ Outros fatores como posicionamento incorreto no leito, pressão insuficiente no balão da cânula traqueal e distensão abdominal, favorecem a broncoaspiração. ${ }^{14}$

Muitas vezes, o concentrado de água que fica armazenado nos circuitos, é retornado freqüentemente aos reservatórios, sendo fácil entender que a colonização ocorre do circuito do respirador para o paciente. Existem vários trabalhos que tentam definir o momento ideal para a troca do circuito, mas nenhum é conclusivo. 15,16,17 0 manuseio inapropriado dos circuitos e as técnicas incorretas de aspiração traqueal constituem fatores de risco. Na UTI, onde este trabalhWo foi desenvolvido, existe a rotina de uso do sistema fechado de aspiração em todos os pacientes submetidos à ventilação mecânica. A credita-se que a diminuição de manipulação da via aérea diminua as complicações pulmonares infecciosas, embora a literatura não seja conclusiva sobre os benefícios do sistema fechado de aspiração. ${ }^{18,19}$

Os grupos tiveram porcentagens similares no tempo de extubação, nos dias de intubação e nas taxas de mortalidade. 0 estudo-trabalho torna-se mais interessante, pois todos os pacientes têm o mesmo diagnóstico (cirrose hepática), enquanto que na literatura, os trabalhos apresentam uma casuística grande, no entanto, com vários tipos de doenças, levando a um questionamento sobre resultados, indicações de intubação, compl icações da ventilação mecânica e a evolução dos pacientes em uma U TI. N este estudo, optou-se por um filtro higroscópico e um hidrofóbico, devido às suas propriedades e capacidade de substituir os sistemas convencionais de umidificação.

Para os pacientes que necessitam de ventilação mecânica, é preciso um sistema que aqueça e produza umidificação do ar, mas a filtração não é realizada. 0 paciente saudável, submetido a qualquer tipo da cirurgia, é completamente diferente do paciente submetido ao transplante de fígado, onde o sistema imunológico está comprometido. 6,20,21,22 N esta casuística, 16 pacientes $(45,7 \%)$ eram pós-operatório de transplante.

0 desempenho do filtro depende do volume corrente. A lguns trabalhos descrevem a oclusão do tubo traqueal em conseqüência de uma umidificação inadequada e também de um volume minuto maior que $10 \mathrm{ml} / \mathrm{kg}^{23,24}$

Existem vários trabalhos que demonstram a eficiência regeneradora de calor e umidade, mas também como barreiras que evitam a passagem das bactérias de um lado ao outro. No entanto, estes trabalhos são laboratoriais. ${ }^{16,25,26,27}$ A credita-se que na prática diária, fatores relacionados ao paciente e à doença de base possam determinar resultados diferentes. No estudo randomizado em pacientes submetidos a ventilação mecânica, foram comparados dois tipos de filtros: um higroscópico e outro hidrofóbico. Concluiu-se que ambos tiveram o poder similar na umidificação, sem diferenças na formação do atelectasias, obstrução da cânula traqueal, colonização e pneumonia relacionada ao respirador. ${ }^{9}$

0 desenvolvimento dos regeneradores de calor e umidade foi uma conquista importante, especialmente para os pacientes imunocomprometidos admitidos na UTI. Com estes filtros, as infecções cruzadas podem ser evitadas, devido à manipulação 
dos circuitos e dos pacientes. Já é bem descrito que o uso de filtro no circuito do ventilador, diminui a colonização. ${ }^{28,29,30,31}$

A quantidade de secreção pulmonar aspirada foi classificada como pequena ( $>1 \mathrm{ml}$ ), moderada ( $>1 \mathrm{ml}$ e $>3 \mathrm{ml}$ ), e grande $(>3 \mathrm{ml}$ ). Essa observação foi importante porque a quantidade de secreção permaneceu constante durante todo o tempo de intubação, não confirmando os trabalhos que descrevem a presença de obstrução de cânula, dificuldade de aspiração e a grande quantidade de secreção nos pacientes admitidos nas UTI. É possível afirmar que estes pacientes receberam cuidados adequados da fisioterapia, pois o volume de secreção traqueal era de pequeno a moderado em cada aspiração e também não houve nenhuma oclusão de cânula por rol has de muco.

A pneumonia produz um aumento na mortalidade e nos custos, devido a uma internação mais longa e o uso de antibióticos. $2,6,32$ Isto se agrava nos pacientes com doença hepática, porque são mais suscetíveis às infecções oportunistas. 0 consumo de massa muscular é conseqüência, não somente da evolução natural da doença, mas também da restrição protéica.Todos estes fatores, adicionados à expectativa do órgão, tornam 0 paciente mais limitado e suscetível às infecções. ${ }^{3} \mathrm{Um}$ outro aspecto que aumenta 0 risco de pneumonia é a freqüência com que os circuitos dos respiradores são trocados. A troca do circuito do respirador a cada 24 ou 48 horas, está associada a um aumento na incidência de pneumonia, que pode ser atribuída à manipulação do circuito e do paciente..$^{33} \mathrm{M}$ ais tarde, demonstraram que os circuitos podem ser trocados por um tempo maior que 48 horas, sem nenhum risco. ${ }^{16,34}$

Para al guns autores, o fato de não trocar os circuitos, ou de trocálos a cada sete dias, não inf luencia na incidência de pneumonia, sendo estas incidências de $24,5 \%$ e $28,8 \%$, respectivamente. ${ }^{35}$ 0 utro autor relatou em estudo prospectivo randomizado, utilizando filtro hidrofóbico e sistema convencional (aquecidos e úmidos), trocados a cada sete dias, que a incidência de pneumonia foi de $6 \%$ e $16 \%$, respectivamente. As taxas de infecção foram significantes no grupo que usou filtro. Observou também uma di minuição no tempo de internação no grupo que usou o filtro, sem nenhuma oclusão da cânula. ${ }^{10}$ No presente estudo, a porcentagem de pneumonia diagnosticada através da broncoscopia foi de $15,8 \%$ no grupo hidrofóbico e $12,5 \%$ no grupo higroscópico.

A aspiração traqueal é importante para a higiene brônquica, mas deve ser feita corretamente. Existem estudos que comparam dois sistemas de aspiração e sugerem que os riscos de contaminação do cateter e pneumonia não são diferentes nos dois grupos. ${ }^{18,19}$ Vantagens, como custo baixo e uma diminuição na contaminação, são atribuídas ao uso do sistema fechado de aspi ração, embora não tenha nenhum trabal ho conclusivo. U ma outra vantagem do uso do sistema fechado de aspiração é a diminuição da manipulação da via aérea e risco da desconexão do circuito. No entanto, são necessários mais trabal hos para se comparar as vantagens e as desvantagens dos sistemas de aspiração.

A pneumonia nosocomial é devida a vários fatores diferentes, e tem um risco maior nos pacientes imunodeprimidos e transplantado com flora alterada. A micro aspiração de sangue ou gástrica, em pacientes sem via aérea artificial e com nível de consciência rebaixado, é fator decisivo na instalação de pneumonia e no prognóstico destes pacientes., ${ }^{7,21}$

A pesquisa de microorganismos nas extremidades dos filtros foi realizada para verificar a capacidade de barreira dos sistemas, ou seja, se permitia a passagem de um lado ao outro. A pesar do número pequeno de casos, é possível dizer que estes dispositivos são capazes de filtrar. A incidência de pneumonia foi igual nos grupos hidrofóbico e higroscópico. A freqüência da contaminação nas extremidades dos filtros não foi estatisticamente significante; provavelmente, os filtros possuem as mesmas características para filtração. Observou-se que na maioria das vezes, a secreção traqueal estava contaminada por pelo menos um agente. A lém disso, a extremidade proximal foi contaminada também pelo mesmo agente da secreção traqueal. A extremidade distal quando foi contaminada, provavelmente tenha sido por ineficiência da filtração. Entretanto, é interessante analisar que o agente colonizador da extremidade distal não persistiu nos dias seguintes. É suposto que a troca dos filtros a cada 24 horas, não permitiu a contaminação dos circuitos dos respiradores.

0 método estatístico empregado facilitou estimar a relação "tempo x variáveis" permitindo a análise de toda informação obtida, independentemente do tempo da observação. A través da anál ise dos gráficos e das tendências lineares do crescimento dos coeficientes lineares médios em relação ao tempo, é possível dizer que, no transporte através da tosse, o filtro higroscópico mantém valores iguais, enquanto que o filtro hidrofóbico mantém uma ten dência decrescente do cresci mento linear, demonstrando que o transporte através da tosse sofreu alteração, resultando uma distância menor. Esta variável foi estatisticamente significante no grupo higroscópico. 0 transporte através dos cílios apresentou uma tendência crescente em ambos os grupos, mostrando uma melhoria de sua condição inicial, provavel mente devido à manutenção das propriedades físicas do muco.

$\mathrm{N}$ a adesão através do ângulo de contato, os crescimentos lineares foram coincidentes e a tendência diminuiu nos dois grupos, isto é, o muco mostrou um acoplamento melhor, provavel mente pela manutenção das propriedades. M as existe uma outra hipótese, talvez o muco tenha ficado mais fluído com o passar dos dias, pela gravidade do doente e pelo maior grau de edema do paciente, e isto foi observado a partir do décimo dia de intubação.

\section{Conclusão}

A incidência de pneumonia foi igual nos grupos higroscópico e hidrofóbico. A contaminação das extremidades foi também similar, no entanto, na extremidade distal ela não se manteve nos dias subseqüentes. A contaminação do circuito ocorre do paciente para o circuito. Os filtros tiveram um comportamento igual no transporte através dos cílios e ângulo de contato. 0 transporte através da tosse foi mais eficiente no grupo higroscópico. 


\section{ABSTRACT}

Objective: To compare the effects of the hydrophobic and hygroscopic filtering (HHF) in the physical properties and transportability of the mucus and lung infection rate. $M$ aterial and $M$ ethod: Thirty five patients included in this study where submitted to mechanical ventilation for more than 24 hours at the Liver Transplant's Intensive Care U nit (ICU). Patients were randomized in two groups immediately after intubation. Group $1(n=19)$ used a B B100 hydrophobic filter, and Group $2(n=16)$ used a Humid-Vent Filter ${ }^{\circledR}$. Light hygroscopic filter. The mean ages of the hydrophobic and hygroscopic groups were $52.4 \pm 13.0$, and $45.5 \pm 16.4$, respectively. Results: Patients had similar fraction of inspired volume of Oxygen, partial pressure of Oxygen / fraction of inspired 0 xygen, minute/volume, to the hydric balance in the previous 24 hours, and to the sodium and bilirubin value. The mucus transportability was assessed through the ciliary transportation and cough. The adherence was measured by the contact angle. The filtering analysis as a bacterial barrier was assessed by the tracheal secretion culture. Condusion: The physical properties of the mucus remained unchanged with the use of the HHF. Nevertheless, the transportation through cough was better in the hygroscopic group. Both H HF can be considered bacterial barriers, and they can reduce the occurrence of the ventilator-related pneumonia.

Keywords: bronchial mucus, heat and moisture regenerator, nosocomial pneumonia, ciliary transportation, mechanical ventilation.

\section{REFERÊNCIAS}

1 Nakagawa NK, Macchione M, Guimaraes ET, King M, Saldiva PH, Lorenzi-Filho G et al. - Effects of heat and moisture exchanger and a heated humidif respiratory mucus in patients undergoing mechanical ventilation. Crit Care Med 2000; 28:312-317.

2 Chastre J, Fagon JT, Soler P, Bornet M, Domart Y, Trouillet JL et al. - Diagnosis of nosocomial bacterial pneumonia in intubated patients undergoing ventilation: comparison of the usefulness of bronchoalveolar lavage and the protected specimen brush. Am J Med 1988; 85:499-506.

3 Duran FG, Piqueras B, Romero M, Carneros JA, De Diego A, Salcedo Met al. - Pulmonary complications following orthotopic liver transplant. Transpl Int 1998;1:S255-9.

4 Eckerbon B \& Lindholm CE - Performance evaluation of six heat and moisture exchangers according to the Draft International Standard (ISSO/DIS9360) Acta Anaesthesiol Scand 1990; 34:404-409.

5 King M, Brock G \& Lundel C - Clearance of mucus by simulated cough. J App. Physiol 1985; 58:1776-1782.

6 Porzecanski I, Bowton DL. Diagnosis and treatment of ventilator-associated pneumonia. Chest. 2006 Aug;130(2):597-604

7 Rosa H, Silverio AO, Perini RF, Arruda CB. Bacterial infection in cirrhotic patients and its relationship with alcohol. Am J Gastroenterol. 2001 May;96(5):1645-6.

8 Thomachot L, Viviand X, Arnaud S, Boisson C \& Martin CD - Comparing two heat and moisture exchangers, one hydrophobic and one higroscopic, on humidifying efficacy and the rate of nosocomial pneumonia. Chest 1998; 114:1383-1389.

9 Kirton OC, Dehaven B, Morgan J, Morejon O \& Civetta J - A prospective, randomized comparison of an in- line heat moisture exchange filter and wire humidifiers: rates of ventilator-associated early onset (community-acquired) or late-onset (hospital-acquired) pneumonia and incidence of tracheal tube occlusion. Chest 1997; 112:1055-1059.

10 Gallagher J, Strangeways JM \& Allt-Graham J - Contamination control in long term ventilation. Anaesthesia 1987; 42: 476-481.

11 CDC . Guideline for prevention of nosocomial pneumonia - MMWR 46: 1 - 79, 1994.

12 Diggle PJ, Liang KY \& Zeger SL - Analysis of Longitudinal Data. Oxford: Oxford Science Publications. 1994.
13 Torres A, Aznar R, Gatell JM, Jimenez P, Gonzalez J, Ferrer A et al. - Incidence, risk and prognosis factors of nosocomial pneumonia in ventilated patients. Am Rev Respir Dis 1990; 142:523-528.

14 Emre S, Sebastian A, Chodoff L, Boccagni P, Meyessrs B, Sheiner PA et al. - Selective decontamination of the digestive tract helps prevent bacterial infections in the early postoperative period after liver transplant. Mt Sinai J Med 1999; 66: 310-313.

15 Djedaini K, Billiard M, Mier L, Le Bourdelles G, Brun P, Markowicz P et al. Changing heat and moisture exchangers every 48 hours rather than 24 hours does not affect their efficacy and the incidence of nosocomial pneumonia. Am J Crit Care Med 1995; 152: 1562-1569.

16 Hess D, Burns E, Romagnoli D \& Kacmarek R M - Circuit change frequency and ventilator-associated pneumonia rates. Chest. 1994; 106:79S. [Abstrat]

17 Kollef MH, Shapiro S D, Fraser VJ, Silver P, Murphyd M, Trovillion E et al. - Mechanical ventilation with or without 7-day circuit changes - A randomized controlled trial. Ann Intern Med. 1995; 123:168-174.

18 Adams DH, Hughes M \& Elliott TS - Microbial colonization of closed-system suction catheters used in liver transplant patients. Intensive Crit Care Nurs 1997; 13:72-76.

19 Crimlisk JT, Paris R, Mcgonagle EG, Calcutt JA \& Farber HW - The closed tracheal suction system: implications for critical care nursing. Dimens Crit Care Nurs 1994; 3:292-300.

20 Duran FG, Piqueras B, Romero M, Caeneros JA, De Diego A, Salcedo M et al. - Pulmonary complications following orthotopic liver transplant. Transpl Int 1998; 1:S255-9.

21 Kondrup J, Nielsen K \& Hamberg O - Nutritional therapy in patients with liver cirrhosis. Eur J Clin Nutr 199246:239-246.

22 Singh N, Gayowski T, Wagener MM \& Marino IR - Pulmonary infiltrates in liver transplant recipients in the intensive care unit. Transplantation 1999; 67:1138-1144.

23 Martin C, Papazian L, Pierrin G, Saux P \& Gouin F- Preservation of humidity and heat of respiratory gases in patients with a minute ventilation greater than $10 \mathrm{~L} / \mathrm{min}$.Critical Care Medicine 199422: 1871-1876.

24 Noguchi H, Takume Y \& Rochi O - A study of humidification in tracheostomized dogs. Br J Anaesth 1973; 45:844-847. 
25 Haas H, Morris JF, Samsom S, Kolbourn JP \& Kim PJ -Bacterial flora of the respiratory tract in chronic bronchitis: Comparison of transtracheal fiberbronchoscopic and oropharyngeal sampling methods. Am Rev Respir Dis 1977; 116:41-47.

26 Holton J \& Webb AR - An evaluation of the microbial retention performance of three ventilator-circuit HME. Intensive Care Med 1994; 20:233-237.

27 Mebius C - Heat and moisture exchangers with bacterial HME: a laboratory evaluation. Acta Anaesthesiol Scand 1992; 36:572-576.

28 Austan F \& Suzukawa M - Humidification method that decreases condensate contamination in ventilator tubing. Heart Lung 2000; 29:56-59.

29 Branson RD, Kenneth DJ, Campbell R, Johnson D \& Porembka D- Humidification in the Intensive Care Unit. Chest 1993;104:1800-1805.

30 Niederman MS, Mantovani R, Schoch P, Papas J \& Fein AM- Patterns and routes of tracheobronchial colonization in mechanically ventilated patients.Chest 1989;95:155-161.

31 Fagon J, Chastre J, Domart Y, Trouillet JL, Pierre J, Darne C et al. - Nosocomial pneumonia in patients receiving continuous mechanical ventilation: prospective analysis of 52 episodes with use of a protect specimen brush and quantitative culture techniques. Am Rev Respir Dis 1989; 139:877-884

32 Craven DE, Kunches LM \& Kilinsky V - Risk factors for pneumonia and fatality in patients receiving continuous mechanical ventilation. Am Rev Respir Dis 1986; 133:792-796.

33 Dreyfuss D, Djedaini K, Weber P, Brun P, Lanore JJ \& Rahmani J - Prospective study of nosocomial pneumonia and of patient and circuit colonization during mechanical ventilation with circuit changes every 48 hours versus no change. Am Rev Respir Dis. 1991; 143:738-743.

34 Kollef MH, Shapiro SD, Boyd V, Silver P, Von Harz B, Trovillion E et al. - A randomized clinical trial comparing an extended-use higroscópico condenser humidifier with heated-water humidification in mechanically ventilated patients. Chest 1998; 113: 759-767.

35 Thomachot L, Leone M, Razzouk K, Antonini F, Vialet R, Martin C. Randomized clinical trial of extended use of a hydrophobic condenser humidifier: 1 vs. 7 days. Crit Care Med. 2002 Jan;30(1):232-7 